\title{
Gluteal muscle composition differentiates fallers from non-fallers in community dwelling older adults
}

Mario Inacio ${ }^{1}$, Alice S Ryan², Woei-Nan Bair ${ }^{1}$, Michelle Prettyman ${ }^{1}$, Brock A Beamer ${ }^{2}$ and Mark W Rogers ${ }^{*}$

\begin{abstract}
Background: Impaired balance, loss of mobility and falls are major problems associated with changes in muscle in older adults. However, the extent to which muscle composition and related performance measures for different lower limb muscles are associated with falls in older individuals is unclear. This study evaluated lower limb muscle attenuation, intramuscular adipose tissue (IMAT) infiltration and muscle performance in older fallers and non-fallers.

Methods: For this cross-sectional study, fifty-eight community dwelling older individuals ( $>65$ years) were classified into fallers ( $n=15)$ or non-fallers $(n=43)$. Computed tomography $(C T)$ was used to determine muscle attenuation and intramuscular adipose tissue (IMAT) of multiple thigh and hip muscles. Muscle performance was assessed with isokinetic dynamometry.

Results: For both groups, Rectus Femoris showed the highest muscle attenuation and lowest IMAT infiltration, and Gluteus Maximus and Gluteus Medius/Minimus muscles had the lowest muscle attenuation and highest IMAT infiltration. Fallers exhibited lower muscle attenuation and higher IMAT infiltration than non-faller participants in most muscles, where the gluteal muscles were the most affected $(p<0.05)$. Fallers also showed a lower peak hip abduction torque $(p<0.05)$. There were significant associations $(r=0.31$ to 0.53$)$ between joint torques and muscle composition, with the strongest associations between Gluteus Medius/Minimus and hip abduction strength.

Conclusions: While fallers were generally differentiated from non-fallers by muscle composition, the most affected muscles were the proximal gluteal muscles of the hip joint accompanied by lower hip abduction strength, which may contribute to impaired balance function and increased risk for falls.
\end{abstract}

Keywords: Older age, Falls, Muscle composition, Muscle performance

\section{Background}

Among older adults, falls and related injuries are major health care problems that diminish quality of living and contribute to increased morbidity and mortality [1-4]. Thus, identifying the characteristics of individuals at higher risk for falls in order to establish effective falls prevention interventions is of major clinical significance [5-8].

Impairments of balance and mobility are important risk factors contributing to age-associated falls $[8,9]$. During everyday activities, protective stepping is normally a commonly used strategy for stabilizing balance

\footnotetext{
*Correspondence: mrogers@som.umaryland.edu

${ }^{1}$ Department of Physical Therapy and Rehabilitation Science, University of

Maryland School of Medicine, Baltimore, MD, USA

Full list of author information is available at the end of the article
}

in response to postural challenges. However, balance control through stepping is problematic for many older individuals, especially in the mediolateral (M-L) direction where vulnerability to imbalance and fall-related injuries may be heightened $[8,10]$. For example, when steps were randomly evoked in 12 different directions by waist-pull postural perturbations, an age-associated reduction in balance recovery effectiveness through stepping was identified particularly for the lateral direction among prospectively identified fallers [11]. A possible reason for diminished lateral balance function may be that older individuals less often engage in activities, such as single limb weight bearing tasks, sideways whole-body movements and other tasks that require vigorous use of the hip abductor-adductor muscles important for lateral 
balance control [10,12-14]. In this regard, older individuals have lower maximum isokinetic hip abduction torque implicated in lateral balance control than younger adults, and these age-related decrements in strength are greater for older individuals at higher risk for falls than for those at lower fall risk $[7,15]$. Although arthritic degeneration with older age can affect muscle composition [16], it is conceivable that the age-related reductions in physical activity patterns may also impact changes in muscle composition that affect balance and mobility particularly involving the mediolateral direction.

Aging brings about changes in skeletal muscle frequently manifested as primary sarcopenia [17]. Primary sarcopenia is characterized by an age-related reduction in muscle mass, strength, quality [18-20] and altered metabolism [21]. These changes often include increased intramuscular adipose tissue (adipose tissue within the epimysium (IMAT)) [22-24] and are associated with physical frailty, loss of mobility and increased risk for fractures $[25,26]$. Furthermore, IMAT infiltration and the density of the skeletal muscle fibers (muscle attenuation) may differentially affect the lower limb musculature $[16,21,27]$.

Although sarcopenia and its associated muscle composition characteristics have received much attention, further clarification is needed concerning their relationship to clinically relevant functional impairments such as diminished muscular performance, balance function and the propensity to fall. Hence, the purpose of this study was to evaluate muscle attenuation and infiltration of IMAT by computed tomography (CT), and muscular performance in older fallers and non-fallers. We hypothesized that the proximal muscles of the hip would demonstrate greatest age-related changes in muscle composition. Furthermore, we anticipated that worse muscle composition would be associated with poorer muscular performance and that elderly fallers would show greater impairments in muscle composition and muscular performance than non-fallers.

\section{Methods}

\section{Subjects}

Participants were recruited mainly by community older adult newspaper advertisements in the Baltimore/ Washington area. In order to recruit a sample of otherwise healthy community dwelling older individuals that would be able to undergo muscle composition and strength testing procedures without major confounding factors, participants aged 65 years or older were screened over the telephone by the recruitment staff, followed by a medical examination performed by a physician geriatrician. Exclusion criteria consisted of the following: 1) cognitive impairment (Folstein Mini Mental Score Exam < 24); 2) sedative use, such as Valium, Xanax or Lunesta; 3) unable to walk independently without the use of an assistive device; 4) any clinically significant functional impairment related to musculoskeletal, neurological, cardiopulmonary, metabolic, or other general medical problem; 5) engaged in a dedicated and structured physical exercise regimen for 3 or more days per week beyond more routine casual or recreational physical activity; and 6) Centers for Epidemiological Studies Depression Survey score greater than 16. The falls assessment was performed during the telephone screen and consisted of the fall history in the 12 months prior to enrollment. A fall was identified based on the criteria adopted by the World Health Organization [28]. This assessment identified 43 individuals $(n=21$ males, $\mathrm{n}=22$ females) to be non-fallers and 15 ( $\mathrm{n}=5$ males, $\mathrm{n}=10$ females) to be fallers. Descriptive information about the study participants is presented in Table 1. All subjects provided written informed consent that was approved by the research ethics committee from the Institutional Review Board of University of Maryland, Baltimore and the Baltimore Veteran's Administration

Table 1 Comparison between non-faller and faller groups on the sample demographics

\begin{tabular}{|c|c|c|c|}
\hline & $\begin{array}{c}\text { Non-fallers } \\
(n=43)\end{array}$ & $\begin{array}{l}\text { Fallers } \\
(n=15)\end{array}$ & $p$ \\
\hline Age (yr) & $74.0 \pm 1.1$ & $73.0 \pm 1.1$ & 0.56 \\
\hline Height $(\mathrm{cm})$ & $166.9 \pm 1.3$ & $165.6 \pm 2.5$ & 0.63 \\
\hline Weight (kg) & $75.1 \pm 2.2$ & $79.0 \pm 5.0$ & 0.42 \\
\hline$B M I\left(k g \cdot m^{-2}\right)$ & $26.7 \pm 0.5$ & $28.2 \pm 1.3$ & 0.21 \\
\hline Fat Mass (kg) & $26.7 \pm 1.1$ & $31.0 \pm 2.5$ & 0.09 \\
\hline Fat-Free Mass (kg) & $45.8 \pm 1.4$ & $47.2 \pm 3.4$ & 0.65 \\
\hline$\%$ Fat Mass & $35.4 \pm 0.9$ & $38.3 \pm 1.7$ & 0.12 \\
\hline \multicolumn{4}{|c|}{ Co-morbidities (\%) } \\
\hline Hypertension & 22.4 & 13.8 & 0.27 \\
\hline Hyperlipidemia & 31.0 & 19.0 & 0.19 \\
\hline Diabetes & 3.4 & 1.7 & 0.56 \\
\hline Coronary disease & 1.7 & 3.4 & 0.56 \\
\hline Edema (lower limbs) & 3.4 & 3.4 & 1.00 \\
\hline Painful joints & 19.0 & 15.5 & 0.65 \\
\hline Osteoarthritis & 24.1 & 13.8 & 0.20 \\
\hline Osteoporosis & 10.3 & 6.9 & 0.53 \\
\hline Depression & 1.7 & 6.9 & 0.18 \\
\hline Incontinence & 1.7 & 1.7 & 1.00 \\
\hline Asthma & 5.2 & 1.7 & 0.32 \\
\hline \multicolumn{4}{|c|}{ Number of reported falls in previous 12 months (\%) } \\
\hline 1 & 0 & 66.7 & \\
\hline 2 & 0 & 26.7 & \\
\hline 3 & 0 & 6.7 & \\
\hline
\end{tabular}

Data is expressed as Mean \pm SEM. Comparisons significant for $p<0.05$. 
Research and Development prior to participation (HP00040282).

\section{Procedures}

This study adopted a single session cross-sectional design where participants underwent a continuous computed tomography (CT) scan (Siemens Somatom Sensation 64 Scanner), from the $2^{\text {nd }}$ lumbar vertebra (L2) to the patella while lying in supine position. From the whole scan, three regions of interest were selected for analysis, abdominal, hip and mid-thigh regions, specifically at the level of the $3^{\text {rd }}$ lumbar vertebrae, $3^{\text {rd }}$ sacral vertebrae and $50 \%$ of femur's length respectively (Figure 1 ). The following muscles were selected: Psoas (PS), Gluteus Maximus (GMax), Gluteus Medius and Minimus (GMm), Vastus Lateralis (VL), Rectus Femoris (RF), Hamstrings (Ham) compartment and Adductor Magnus and Longus (Add). Muscle cross section area (CSA), Intramuscular adipose tissue (IMAT) content and muscle attenuation were determined with MIPAV (Medical Image Processing, Analysis and Visualization, v 7.0, NIH) analysis software by tracing the fascia around the selected muscles. Scanning and analysis procedures have been previously reported in detail [24], with CT data expressed as cross-sectional area of tissue $(\mathrm{cm} 2)$, and using Hounsfield units (HU) for muscle area between 30 to $80 \mathrm{HU}$, fat as -190 to $-30 \mathrm{HU}$, and low density lean tissue as 0-29 HU [24]. In addition, the IMAT content was normalized to the respective muscle's size (normalized IMAT $\left.=100 * \frac{\mathrm{IMAT}}{\mathrm{CSA}}\right)$ and expressed as a percentage of the muscle's CSA $\left(\mathrm{cm}^{2}\right)$ [29].

Height $(\mathrm{cm})$ and weight $(\mathrm{kg})$ were measured to calculate body mass index $\left(B M I, \mathrm{~kg} / \mathrm{m}^{2}\right)$. Fat mass and lean tissue mass (fat-free mass = lean + bone) were determined by Dual-energy X-ray Absorptiometry (Prodigy, LUNAR Radiation Corp., Madison, WI). A trained radiology technician performed all DXA scans. Our past referenced CV's for total body percent fat, fat tissue mass, lean tissue mass, and $\mathrm{BMC}$ are $1.4 \%, 1.4 \%, 0.7 \%$, and $0.4 \%$, respectively [30].

Isokinetic strength testing (Biodex System 4, Biodex Medical Systems, Shirley, NY) performed by blinded trained research staff involved performing bilateral concentric contractions at $60^{\mathrm{o} / \mathrm{s}}$ for knee flexion-extension (seated), hip flexion-extension (supported standing), and hip abduction-adduction (supported standing). An external stabilization frame was used to minimize extraneous body movements while standing similar to previous study [7]. Knee flexion-extension was performed from $90^{\circ}$ to $5^{\circ}$ of knee flexion, hip flexion-extension was performed from $0^{\circ}$ to $45^{\circ}$ of hip flexion and hip abductionadduction was performed from $0^{\circ}$ to $30^{\circ}$ of hip abduction. Due to a change in isokinetic measurement system used during the study and to procedural errors on some of the tests, the number of participants used in the analysis of the isokinetic performance variables was 12 non-fallers and 10 fallers. All peak torque data were normalized to

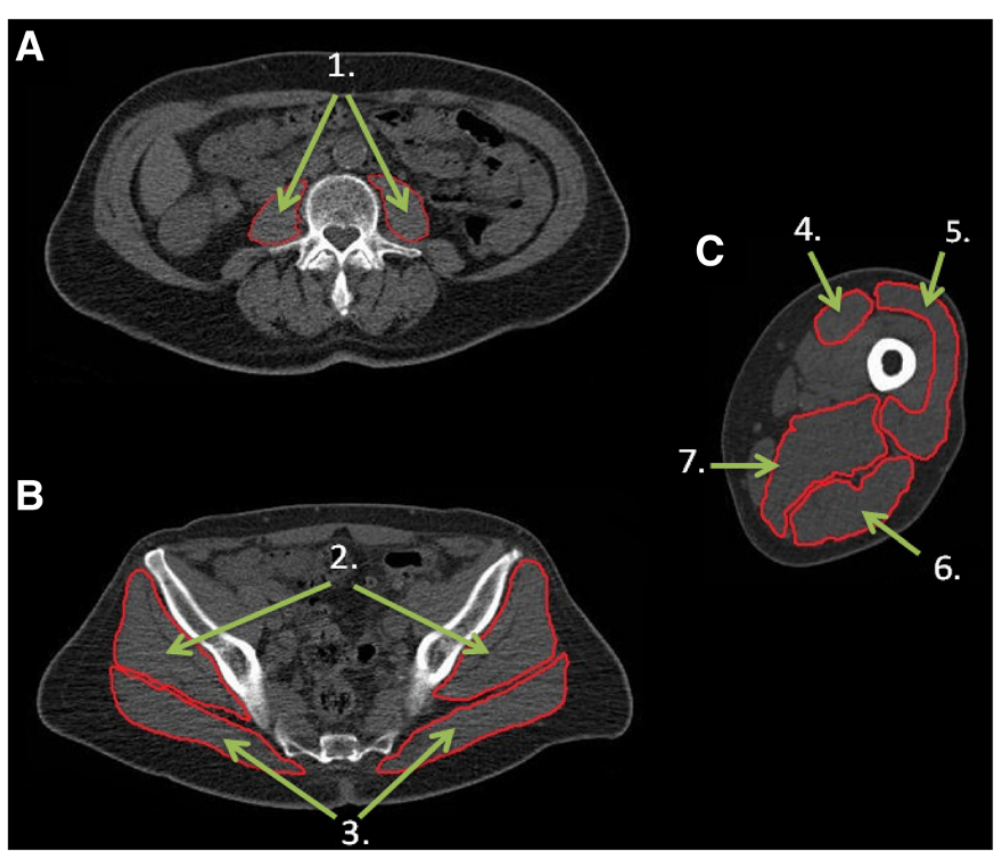

Figure 1 Representative example of computed tomography (CT) scans from an older adult showing A) abdominal scan; B) hip scan; C) thigh scan. 1. Psoas; 2. Gluteus Medius and Minimus; 3. Gluteus Maximus; 4. Rectus Femoris; 5. Vastus Lateralis; 6. Hamstrings compartment; 7. Adductor Magnus and Longus. 
height and weight of each subject using the following equation:

$$
\text { Normalized Torque }=\frac{\text { Peak Torque }}{\text { Height } * \text { Weight }}
$$

\section{Statistical analyses}

Statistical analyses were performed using SPSS (v.17) statistical software. Considering the muscle composition measures did not have a normal distribution (ShapiroWilk test, $p<0.05$ ), comparisons between non-fallers and fallers groups on muscle attenuation, IMAT and CSA were performed by a Mann-Whitney U test. Betweenmuscle comparisons on each individual group were performed by a Kruskal-Wallis one-way analysis of variance, followed by a series of Mann-Whitney U tests to test for pairwise comparisons where Bonferroni correction for multiple comparisons was applied. Muscle performance measures were normally distributed (Shapiro-Wilk test, $p>$ $0.05)$, thus between-group comparisons of peak joint torque were performed by independent samples t-test. Associations between joint torque and muscle composition data were performed using Pearson's correlation. Significance was set at $p<0.05$.

\section{Results}

Age, height, weight and BMI were not significantly different between groups. Approximately two thirds of the faller group experienced a single fall in the previous 12 months while the remainder of the group had two or more falls (Table 1). In accordance with the exclusion criteria, the existing co-morbidities are presented in Table 1. Due to the different gender ratio in the groups, gender was used as a covariate to assess any potential gender differences on the different groups. The results from this initial analysis of covariance showed that both genders were similar in every muscle and fall classification group for percent IMAT and muscle attenuation, with only the exceptions of Psoas and Vastus Lateralis muscles in the non-faller group for normalized IMAT (see Additional file 1, Table A1 for further details). Considering that, in general, gender appeared not to impact the present results, the entire data set was used in the analyses and described below.

\section{Between-muscle comparisons}

Multiple significant differences were found between muscle groups (see Additional file 2, Table A2 for the complete analysis). Overall, Gluteus Maximus muscle attenuation was lower than all of the other muscles $(p<$ 0.0024), whereas Rectus Femoris had the highest muscle attenuation $(p<0.0024)$. In the non-faller group, Gluteus Medius/Minimus and Adductors had significantly higher muscle attenuation than Gluteus Maximus but lower values than all other muscles $(p<0.0024)$. In the faller group, Gluteus Medius/Minimus, Vastus Lateralis and Adductors had similar muscle attenuation $(p>$ 0.0024), which was greater than Gluteus Maximus but less than all of the other muscles $(p<0.0024)$. The percentage of IMAT infiltration was highest for Gluteus Maximus $(p<$ $0.0024)$ and lowest for Rectus Femoris $(p<0.0024)$ in both groups. In the non-faller group, the relative adipose infiltration was similar between Gluteus Medius/Minimus and Gluteus Maximus $(p>0.0024)$. In the faller group, Gluteus Medius/Minimus and Psoas muscles had similar relative adipose tissue infiltration $(p>0.0024)$ that was significantly less than that for Gluteus Maximus, but more than for the other muscles $(p<0.0024)$.

\section{Between-group comparisons}

Muscle CSA was similar between groups for all of the muscles $(p>0.05)$. However, the non-faller group had higher muscle attenuation values for most muscles compared with the faller group ( $p<0.05$, Figure 2$)$. Likewise, percent IMAT in the Psoas, Rectus Femoris, Hamstrings, Adductors, Gluteus Maximus and Gluteus Medius/Minimus was higher in the faller group $(p<0.05$, Figure 3$)$. Normalized peak joint torque differed between the groups only for hip abduction, which was greater for the nonfallers $(p<0.05$, Table 2$)$.

\section{Associations between joint torque and muscle composition}

Hip abduction torque was inversely associated with Gluteus Medius/Minimus percent IMAT infiltration $(r=-0.49, p<$ $0.01)$ and positively associated with muscle attenuation $(r=$ $0.53, p<0.01$ ). Hip extension torque was inversely associated with Gluteus Maximus percent IMAT infiltration $(r=-0.39, p<0.05)$. There was a trend for an association between hip extension torque and Gluteus Maximus muscle attenuation $(r=0.31, p=0.056)$. Similarly, hip flexion torque was inversely associated with Psoas percent IMAT infiltration $(r=-0.46, p<0.01)$ and positively associated with muscle attenuation $(r=0.34$, $p<0.05)$. Knee flexion torque was associated with Hamstrings muscle attenuation $(r=0.41, p<0.01)$, but not with IMAT infiltration. Additionally, the normalized knee extension torque was not associated with any of the muscle composition measures for the Vastus Lateralis and Rectus Femoris. A similar lack of associations was observed between hip flexion torque and Rectus Femoris, as well as between hip adduction torque and Adductors.

\section{Discussion}

This was among the first studies to investigate whether age-related changes in lower limb muscle composition 


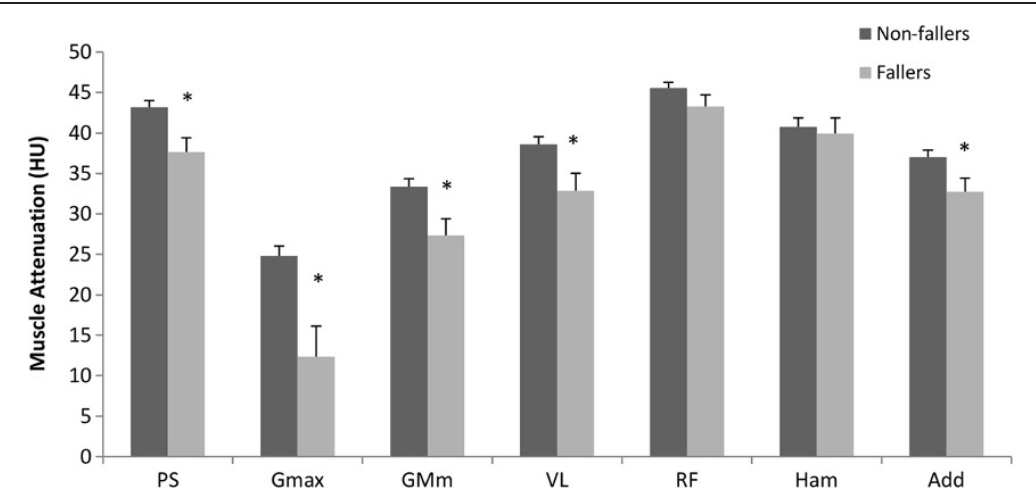

Figure 2 Comparison between non-faller and faller groups for muscle attenuation (HU). Data expressed as Mean \pm SEM. * indicates significant difference $(p<0.05)$.

and performance differentiated between faller and nonfaller cohorts of older adults who were reportedly not residing in community care or other health care settings, and have not scored zero in any item of the Instrumental Activities of the Daily Living (IADL) impairment screening tool. Twenty-six percent of the participants reported falling one or more times during the yearlong monitoring period prior to testing. This incidence of falls generally resembled past studies $[3,7,8,31]$.

\section{Between-muscle comparison}

As hypothesized, muscle composition changes differed between lower limb muscles. Muscle attenuation analysis revealed several differences between the selected muscles across the two groups, where Rectus Femoris had the highest values and Gluteus Maximus and Gluteus Medius/Minimus showed the lowest values. These data suggested that the gluteal muscles may be particularly susceptible to composition changes with advancing age as evidenced by lower muscle density and reduced force generating capacity compared with other muscles of the lower limb [20].

Considering that muscle attenuation is inherently related to muscular adipose content [32], our findings for the adipose tissue infiltration were not surprising. In both groups, when adiposity was expressed relative to the specific muscle's cross-sectional area, we observed that Gluteus Maximus and Gluteus Medius/Minimus showed the highest relative infiltration of adipose tissue. Although previous reports have demonstrated age-related differences in composition between different muscles $[16,21,27]$, we found the most prominent composition changes for the proximal muscles of the hip. This novel finding has clinical relevance as it indicates that disproportionate changes may occur involving the proximal hip musculature. These changes may not only affect overall muscle function, but may especially impact the ability to stabilize standing balance in the frontal plane. In this connection, osteoarthritis is associated with hip muscle dysfunction and impaired balance [33,34]. Although only a

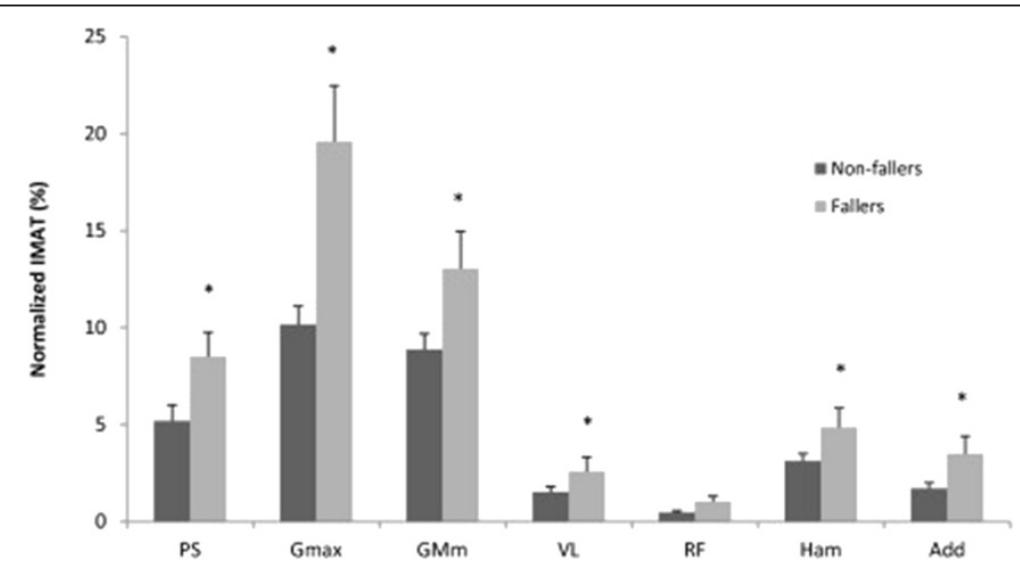

Figure 3 Comparison between non-faller and faller groups for normalized intramuscular adipose tissue (IMAT) (\%). Data expressed as Mean \pm SEM. * indicates significant difference $(p<0.05)$. 
Table 2 Comparison between non-faller and faller groups on normalized peak isokinetic torque ( $\left.\mathrm{N} . \mathrm{Kg}^{-1}\right)$

\begin{tabular}{lccc}
\hline & $\begin{array}{c}\text { Non-fallers } \\
(\boldsymbol{n}=\mathbf{1 2})\end{array}$ & $\begin{array}{c}\text { Fallers } \\
(\boldsymbol{n}=\mathbf{1 0})\end{array}$ & $\boldsymbol{p}$ \\
\hline Knee Extension & $0.71 \pm 0.03$ & $0.61 \pm 0.03$ & 0.06 \\
Knee Flexion & $0.31 \pm 0.01$ & $0.32 \pm 0.02$ & 0.64 \\
Hip Extension & $0.37 \pm 0.03$ & $0.36 \pm 0.03$ & 0.73 \\
Hip Flexion & $0.59 \pm 0.02$ & $0.54 \pm 0.03$ & 0.12 \\
Hip Abduction & $0.53 \pm 0.02$ & $0.41 \pm 0.03$ & $* 0.01$ \\
Hip Adduction & $0.22 \pm 0.02$ & $0.22 \pm 0.02$ & 0.90 \\
\hline
\end{tabular}

Data is expressed as Mean \pm SEM. *indicates significant difference $(p<0.05)$.

small percentage of our study participants reported some type of mild osteoarthritis that did not affect daily living activities, it is possible that even asymptomatic osteoarthritic changes in the hip joints may be contributing to the observed muscle composition changes.

\section{Between-group comparison}

Muscle CSA was not different between the groups for any of the muscles, and therefore does not likely confound the interpretation of the results. Nonetheless, even though the classical measurement of sarcopenia (muscle size) was not different between the groups, other muscle composition changes, such as skeletal muscle density and adipose infiltration, may be different between individuals and potentially impact performance and functional activities.

To our knowledge, this study has identified previously unreported differences in muscle composition between older fallers and non-fallers. Fallers had lower muscle attenuation in most muscle groups, with Gluteus Maximus and Gluteus Medius/Minimus showing the greatest reductions in muscle density. In addition, normalized IMAT was higher in the faller group for most of the muscle groups but especially for Gluteus Maximus and Gluteus Medius/Minimus. In particular, IMAT infiltration in Gluteus Maximus of the fallers was double that of the non-fallers. These findings differentiating between fallers and non-fallers are especially noteworthy because they were not just attributable to increased BMI as these were non-obese older adults. While several muscle composition measures identified differences between the groups, these differences were only reflected in impaired performance for the hip abductor muscles, where fallers were weaker than non-fallers.

\section{Associations between joint torque and muscle composition}

Because of the inherent inverse relationship between IMAT infiltration and muscle attenuation, it was expected that joint torque and muscle attenuation would be positively associated, while torque and adipose infiltration would be negatively associated. These results indicated that the muscle composition changes were not only greater among fallers but were also associated with their poorer muscle performance.

When considered collectively, the findings indicated that changes in lower limb muscle composition in general, and of the gluteal muscles in particular, distinguished between older fallers and non-fallers. It is widely recognized that sarcopenic changes are related to loss of muscle mass, reduced muscle strength, physical frailty, and increased risk of fracture $[19,26]$. Such changes have also been associated with loss of mobility [25,27,35,36] and limitations in balance function [37]. For example, increased muscle fat infiltration has been shown to be related to reduced knee extensor muscle strength, as well as future mobility limitations among well-functioning older individuals [25,36]. Similarly, IMAT accumulation in the thigh has been identified as a predictor of 6 minute walk distance, stair ascent/descent, and Timed Up and Go time [35]. Among older adults with a history of back pain, increased trunk muscle fat infiltration predicted diminished functional capacity especially for balance outcomes [38]. Hence, the present findings have implications for mobility disability related to falls among community dwelling older individuals.

With respect to balance function, protective stepping is normally a primary strategy for recovering balance in the everyday environment that becomes more prevalent and problematic for those at greater risk for falls, especially in the mediolateral direction $[8,10,11]$. An age-associated reduction in the maximum hip abductor-adductor muscle torque generating capacity has been linked with impaired protective stepping in the lateral direction and prospective risk for falls $[7,14]$. Although the non-fallers in this study demonstrated greater torque production than fallers only for hip abduction, we generally found significant associations between peak joint torques and muscle composition. The strongest associations were found for Gluteus Medius/ Minimus muscles that contribute to hip abduction torque and are important for lateral balance stability. We propose that the present results may be indicative of an underlying mechanism contributing to functional deficits in frontal plane balance stability experienced by older individuals at greater risk for falls $[37,39]$. The substantial composition changes for Gluteus Maximus among the faller group also has relevance to lateral stability during gait where older adults have demonstrated an increased reliance on Gluteus Maximus (hip and back extensor muscle) contributions to controlling mediolateral balance when compared to younger adults [40]. Additionally, as a primary hip extensor muscle, Gluteus Maximus IMAT infiltration could contribute to impaired recovery from a trip or slip while walking via forward or backward protective stepping [37,39,41]. 
From a rehabilitation perspective, previous studies have shown that muscle composition can be improved with exercise training and nutrition in older individuals [23,42-46]. Therefore, the present results may help to guide exercise interventions that target proximal hip muscle composition and performance to enhance balance and mobility, two important factors in fall prevention.

Among the limitations of this study is the extent to which the results may be more generally applicable to older individuals beyond community dwelling older adults who are relatively healthy and functionally independent. Thus, frail older people with other morbidities affecting functional capacity may present different muscle composition profiles than those identified here. Additionally, although we found significant associations between muscle composition and muscular performance, the cross-sectional design of the study has a limited potential for inferring causality. A small and similar percentage of individuals in both groups reported experiencing some type of osteoarthritis, which has been associated with reduced muscle composition and quality [16,47]. Although unlikely, it is possible that it may be impacting the outcome measurements. Furthermore, the statistical power for the strength measurements may have also been affected due to the smaller sample size used in this particular test. Another limitation is that we did not account for possible differences in participants' physical activity levels. However, the impact of this limitation may have been minimized as none of the subjects were participating in a regular vigorous exercise program. The smaller sample size in the faller group was a further limitation of the study. However, the incidence of falls involving $26 \%$ of our subjects generally resembles prior studies $[3,7,8,31]$. It is also acknowledged that the retrospective self-report for falls may have underestimated their true incidence compared with a calendar method [48]. Nevertheless, self-report remains as an important source of information about falls occurring in the community and our present data may bolster the notion that individuals self-reporting falls are physiologically distinct from those who do not.

\section{Conclusions}

In summary, the findings indicated that age-related changes in muscle composition are not equivalent throughout the lower limb musculature. While fallers were differentiated from non-fallers by greater IMAT infiltration and greater reduction in muscle attenuation of several muscles, the most affected muscles were Gluteus Maximus, Medius and Minimus. These regionally disparate changes in muscle composition may influence directional changes in lateral balance function, and possibly, successfully recovering from a trip while walking or backward falls.

\section{Additional files} Additional file 1: Table A1. Between-gender comparisons in the
non-faller and faller groups.

Additional file 2: Table A2. Between-muscle comparisons in the non-faller and faller groups.

Competing interests

The authors have no conflicts of interest related to the content of this manuscript.

\section{Acknowledgments}

The authors acknowledge the Claude D. Pepper Older Americans Independence Center, University of Maryland School of Medicine, Baltimore, MD, USA, and the assistance of D. Yungher, J. Morgia and K. Riddle. The helpful comments of Dr. Odessa Addison on a draft manuscript are gratefully acknowledged. This work was supported by the National Institute on Aging at the National Institutes of Health (R01AG029510, P30AG028747), Claude D Pepper - Older Americans Independence Center Grant (OAIC) NIH/NIA P30 AG028747, University of Maryland Advanced Neuromotor Rehabilitation Research Training (UMANRRT) Program, supported by the National Institute of Disability and Rehabilitation Research post-doctoral training grant (H133P100014) and a VA Research Career Scientist Award to Alice S. Ryan.

\section{Authors' contributions}

MI carried out data collection and analysis, and drafted the manuscript. AR helped to draft the manuscript and with CT analysis or data analysis. WB and BB participated in the design of the study. MP participated in the design and coordination. MR conceived the study and helped to draft the manuscript. All authors read and approved the final manuscript.

\section{Author details}

'Department of Physical Therapy and Rehabilitation Science, University of Maryland School of Medicine, Baltimore, MD, USA. ²Division of Gerontology and Geriatric Medicine, Baltimore VAMC GRECC and Department of Medicine, University of Maryland School of Medicine, Baltimore, MD, USA.

Received: 10 September 2013 Accepted: 13 March 2014 Published: 25 March 2014

\section{References}

1. Alexander BH, Rivara FP, Wolf ME: The cost and frequency of hospitalization for fall-related injuries in older adults. Am J Public Health 1992, 82(7):1020-1023.

2. Englander F, Hodson TJ, Terregrossa RA: Economic dimensions of slip and fall injuries. J Forensic Sci 1996, 41(5):733-746.

3. Hornbrook MC, Stevens VJ, Wingfield DJ, Hollis JF, Greenlick MR, Ory MG: Preventing falls among community-dwelling older persons: results from a randomized trial. Gerontologist 1994, 34(1):16-23.

4. Stevens J: Fatalities and injuries from falls among older adults-United States, 1993-2003 and 2001-2005. MMWR Morb Mortal Wkly Rep 2006, 55(45):1221-1224.

5. Brauer SG, Burns YR, Galley P: A prospective study of laboratory and clinical measures of postural stability to predict community-dwelling fallers. J Gerontol A Biol Sci Med Sci 2000, 55(8):M469-M476.

6. De Rekeneire N, Visser M, Peila R, Nevitt MC, Cauley JA, Tylavsky FA, Simonsick EM, Harris TB: Is a fall just a fall: correlates of falling in healthy older persons. The Health, Aging and Body Composition Study. J Am Geriatr Soc 2003, 51(6):841-846.

7. Hilliard MJ, Martinez KM, Janssen I, Edwards B, Mille ML, Zhang Y, Rogers MW: Lateral balance factors predict future falls in community-living older adults. Arch Phys Med Rehabil 2008, 89(9):1708-1713.

8. Maki BE, Holliday PJ, Topper AK: A prospective study of postural balance and risk of falling in an ambulatory and independent elderly population. J Gerontol 1994, 49(2):M72-M84.

9. Tinetti ME, Speechley M, Ginter SF: Risk factors for falls among elderly persons living in the community. N Engl J Med 1988, 319(26):1701-1707.

10. Mille ML, Johnson ME, Martinez KM, Rogers MW: Age-dependent differences in lateral balance recovery through protective stepping. Clin Biomech (Bristol, Avon) 2005, 20(6):607-616. 
11. Mille M, Hilliard M, Martinez K, Zhang Y, Edwards B, Rogers M: One step, two steps, three steps more ... directional vulnerability to falls in community dwelling older people. J Gerontol A Bio/ Sci Med Sci 2013, 68(12):1540-1548.

12. DiPietro L: Physical activity in aging: changes in patterns and their relationship to health and function. J Gerontol A Biol Sci Med Sci 2001, 56 Spec No 2:13-22.

13. Gottschall JS, Okita N, Sheehan RC: Muscle activity patterns of the tensor fascia latae and adductor longus for ramp and stair walking. $J$ Electromyogr Kinesiol 2012, 22(1):67-73.

14. Rogers MW, Mille ML: Lateral stability and falls in older people. Exerc Sport Sci Rev 2003, 31(4):182-7.

15. Johnson ME, Mille ML, Martinez KM, Crombie G, Rogers MW: Age-related changes in hip abductor and adductor joint torques. Arch Phys Med Rehabil 2004, 85(4):593-7.

16. Rasch A, Bystrom AH, Dalen N, Berg HE: Reduced muscle radiological density, cross-sectional area, and strength of major hip and knee muscles in 22 patients with hip osteoarthritis. Acta Orthop 2007, 78(4):505-10.

17. Cruz-Jentoft AJ, Baeyens JP, Bauer JM, Boirie Y, Cederholm T, Landi F, Martin FC, Michel JP, Rolland Y, Schneider SM, Topinkova E, Vandewoude M, Zamboni M: Sarcopenia: European consensus on definition and diagnosis: Report of the European Working Group on Sarcopenia in Older People. Age Ageing 2010, 39(4):412-23.

18. Cawthon PM, Fox KM, Gandra SR, Delmonico MJ, Chiou CF, Anthony MS, Sewall A, Goodpaster B, Satterfield S, Cummings SR, Harris TB: Do muscle mass, muscle density, strength, and physical function similarly influence risk of hospitalization in older adults? J Am Geriatr Soc 2009, 57(8):1411-9.

19. Lang T, Cauley JA, Tylavsky F, Bauer D, Cummings S, Harris TB: Computed tomographic measurements of thigh muscle cross-sectional area and attenuation coefficient predict hip fracture: the health, aging, and body composition study. J Bone Miner Res 2010, 25(3):513-9.

20. Goodpaster BH, Carlson CL, Visser M, Kelley DE, Scherzinger A, Harris TB, Stamm E, Newman AB: Attenuation of skeletal muscle and strength in the elderly: The Health ABC Study. J Appl Physiol 2001, 90(6):2157-65.

21. Nakagawa $Y$, Hattori M, Harada K, Shirase R, Bando M, Okano G: Age-related changes in intramyocellular lipid in humans by in vivo H-MR spectroscopy. Gerontology 2007, 53(4):218-23.

22. Cree MG, Newcomer BR, Katsanos CS, Sheffield-Moore M, Chinkes D, Aarsland A, Urban R, Wolfe RR: Intramuscular and liver triglycerides are increased in the elderly. J Clin Endocrinol Metab 2004, 89(8):3864-71.

23. Marcus RL, Addison O, Kidde JP, Dibble LE, Lastayo PC: Skeletal muscle fat infiltration: impact of age, inactivity, and exercise. J Nutr Health Aging 2010, 14(5):362-6.

24. Ryan AS, Nicklas BJ: Age-related changes in fat deposition in mid-thigh muscle in women: relationships with metabolic cardiovascular disease risk factors. Int J Obes Relat Metab Disord 1999, 23(2):126-32.

25. Visser M, Goodpaster BH, Kritchevsky SB, Newman AB, Nevitt M, Rubin SM, Simonsick EM, Harris TB: Muscle mass, muscle strength, and muscle fat infiltration as predictors of incident mobility limitations in wellfunctioning older persons. J Gerontol A Biol Sci Med Sci 2005, 60(3):324-33.

26. Schafer AL, Vittinghoff E, Lang TF, Sellmeyer DE, Harris TB, Kanaya AM, Strotmeyer ES, Cawthon PM, Cummings SR, Tylavsky FA, Scherzinger AL, Schwartz AV: Fat infiltration of muscle, diabetes, and clinical fracture risk in older adults. J Clin Endocrinol Metab 2010, 95(11):E368-72.

27. Tuttle $L J$, Sinacore DR, Mueller MJ: Intermuscular adipose tissue is muscle specific and associated with poor functional performance. J Aging Res 2012, 2012:1-7.

28. W.H.O: Falls; 2012. Available from: http://www.who.int/violence_injury prevention/other_injury/falls/en/index.html.

29. Bouche KG, Vanovermeire O, Stevens VK, Coorevits PL, Caemaert JJ, Cambier DC, Verstraete K, Vanderstraeten GG, Danneels LA: Computed tomographic analysis of the quality of trunk muscles in asymptomatic and symptomatic lumbar discectomy patients. BMC Musculoskelet Disord 2011, 12:65.

30. Ryan AS, Nicklas BJ, Dennis KE: Aerobic exercise maintains regional bone mineral density during weight loss in postmenopausal women. J Appl Physiol (1985) 1998, 84(4):1305-10.

31. Lord SR, Rogers MW, Howland A, Fitzpatrick R: Lateral stability, sensorimotor function and falls in older people. J Am Geriatr Soc 1999, 47(9):1077-81.

32. Goodpaster BH, Thaete FL, Kelley DE: Composition of skeletal muscle evaluated with computed tomography. Ann N Y Acad Sci 2000, 904:18-24.
33. Hatton AL, Sportsphysio JL, Brauer SG, Clark RA, Crossley KM: Dynamic single-leg balance performance is impaired in individuals with hip chondropathy. Arthritis Care Res (Hoboken) 2013. doi:10.1002/acr.22193.

34. Sims KJ, Richardson CA, Brauer SG: Investigation of hip abductor activation in subjects with clinical unilateral hip osteoarthritis. Ann Rheum Dis 2002, 61(8):687-92.

35. Marcus RL, Addison O, Dibble LE, Foreman KB, Morrell G, Lastayo P: Intramuscular adipose tissue, sarcopenia, and mobility function in older individuals. J Aging Res 2012, 2012:629-637.

36. Schaap LA, Koster A, Visser M: Adiposity, muscle mass, and muscle strength in relation to functional decline in older persons. Epidemiol Rev 2012 [Epub ahead of print].

37. Grabiner MD, Bareither ML, Gatts S, Marone J, Troy KL: Task-specific training reduces trip-related fall risk in women. Med Sci Sports Exerc 2012, 44(12):2410-4.

38. Hicks GE, Simonsick EM, Harris TB, Newman AB, Weiner DK, Nevitt MA, Tylavsky FA: Trunk muscle composition as a predictor of reduced functional capacity in the health, aging and body composition study: the moderating role of back pain. J Gerontol A Biol Sci Med Sci 2005, 60(11):1420-4

39. Owings TM, Pavol MJ, Grabiner MD: Mechanisms of failed recovery following postural perturbations on a motorized treadmill mimic those associated with an actual forward trip. Clin Biomech (Bristol, Avon) 2001, 16(9):813-9.

40. Lim YP, Lin YC, Pandy MG: Muscle function during gait is invariant to age when walking speed is controlled. Gait Posture 2013, 38(2):253-9.

41. Schulz BW, Ashton-Miller JA, Alexander NB: Compensatory stepping in response to waist pulls in balance-impaired and unimpaired women. Gait Posture 2005, 22(3):198-209.

42. Goodpaster BH, Chomentowski P, Ward BK, Rossi A, Glynn NW, Delmonico MJ, Kritchevsky SB, Pahor M, Newman AB: Effects of physical activity on strength and skeletal muscle fat infiltration in older adults: a randomized controlled trial. J Appl Physiol 2008, 105(5):1498-503.

43. Prior SJ, Joseph LJ, Brandauer J, Katzel LI, Hagberg JM, Ryan AS: Reduction in midthigh low-density muscle with aerobic exercise training and weight loss impacts glucose tolerance in older men. J Clin Endocrinol Metab 2007, 92(3):880-6.

44. Ryan AS, Nicklas BJ, Berman DM, Dennis KE: Dietary restriction and walking reduce fat deposition in the midthigh in obese older women. Am J Clin Nutr 2000, 72(3):708-13.

45. Sipila S, Suominen $\mathrm{H}$ : Effects of strength and endurance training on thigh and leg muscle mass and composition in elderly women. J Appl Physiol 1995, 78(1):334-40.

46. Taaffe DR, Henwood TR, Nalls MA, Walker DG, Lang TF, Harris TB: Alterations in muscle attenuation following detraining and retraining in resistance-trained older adults. Gerontology 2009, 55(2):217-23.

47. Conroy MB, Kwoh CK, Krishnan E, Nevitt MC, Boudreau R, Carbone LD, Chen $H$, Harris TB, Newman AB, Goodpaster BH: Muscle strength, mass, and quality in older men and women with knee osteoarthritis. Arthritis Care Res (Hoboken) 2012, 64(1):15-21.

48. Mackenzie L, Byles J, D'Este C: Validation of self-reported fall events in intervention studies. Clin Rehabil 2006, 20(4):331-9.

\section{doi:10.1186/1471-2318-14-37}

Cite this article as: Inacio et al:: Gluteal muscle composition differentiates fallers from non-fallers in community dwelling older adults. BMC Geriatrics 2014 14:37. 